18 - 20 September, 2020

\title{
Being a Hakka in Museums
}

\author{
Bing Wang \\ School of Fine Art, History of Art \& Cultural Studies, University of Leeds
}

\begin{abstract}
Hakka 客家, 'the Guest people', has long been considered as a distinguished minxi (subbranches within a nation) of the Han Chinese when Professor Luo Xianglin published An Introduction to the Study of the Hakkas in Its Ethnic, Historical, and Cultural Aspects in 1933. Hakka themselves welcomed this recognition and were proud to be subsumed into the Han majority. Thus, Hakka have gradually been silenced and actively silenced their own Hakka identity under the influence of Han nationalism by the Nationalist government in order to fight against the evasion from the Western imperial power. Moreover, after the establishment of the People's Republic of China in 1949, the ethnic policies decided that only fifty six 'nationalities' (ethnic groups) were officially recognised. Hakka was not included but subsumed into the Han permanently. However, the concept of Han is anything but primordial and the cultural diversities within are gradually disappearing due to China's ethnic policies.
\end{abstract}

Since 1980s, there was a revival of Hakka studies and led to an increase in Hakka museums and heritage sites. Hakka culture was celebrated and welcomed. Under this circumstance, as a Hakka descendant myself, I found this confusing in term of my own Hakka identity. Thus, I adopt the autoethnographic approach to work with my own visits to a few Hakka museums and heritage sites to explore my feelings of being a Hakka in this contemporary world.

My study benefits from this methodology to enable me as a researcher to examine the nuances of Hakka identity.

Keywords: Hakka, Autoethnography, Museum, Ethnicity, Identity 